\title{
Erratum: Using an atom interferometer to infer gravitational entanglement generation [PRX Quantum 2, 030330 (2021)]
}

\author{
D. Carney, H. Müller, and J.M. Taylor
}

(Received 2 November 2021; published 11 February 2022)

DOI: 10.1103/PRXQuantum.3.010902

The original version of this paper contains a technical error which changes some of the conclusions. The essence of this error was brought to our attention by Streltsov, Pedernales, and Plenio. Here we explain the error, examine its consequences, and suggest methods to overcome the resulting weakness in the proposed experiment.

In brief, some of the statements in the proof of our theorem 1 do not follow from the assumption (c), but rather require a modified version (c') detailed below. Because of this, it is possible to construct a channel on the qubit-oscillator system which satisfies all of our original requirements (time-translation invariance, preservation of $\sigma_{z}$, and separable), which nevertheless exhibits (partial) collapse-and-revival dynamics. Thus observation of a collapse-and-revival signal alone is insufficient to conclude that the underlying channel is capable of entanglement generation.

Our paper should be amended as follows: replace assumption (c) of the Theorem with (c'), detailed below. Replace use of "separable channel" with "Lindblad-form separable channel" (as defined in (c')) wherever it occurs in the paper. We also suggest adding a new line to Table II indicating that non-entangling theories that are not Lindblad-form separable will still show revival, and thus must be tested with more advanced techniques.

Before moving to the details, we note that the experiment proposed in our paper may still be able to rule out the types of counterexamples constructed here. This is because these models necessarily induce additional noise to the experiment, which will partially destroy the revival signature. Seeing revival above this loss would rule out such a non-entangling model. We also emphasize that the corrected version of the main arguments are still sufficient to rule out a wide class of models, namely those in which gravity arises as a Lindblad-separable, semi-classical stochastic force, and we provide a short example of such a model for reference.

Formal issue. The central technical result of the paper is stated as a theorem about two-body channels $L$ obeying a certain separability criterion. This was assumption (c) in the paper, viz.

(c) $L$ is a separable channel: all of its Krauss operators are simple products. In particular, this means that any initial separable (non-entangled) state evolves to a separable state: $\rho(t)=L_{t}[\rho(0)]$ is separable for all separable initial states $\rho(0)$.

Given such a separable Krauss representation exists, say $L[\rho]=\sum_{i} L_{i} \rho L_{i}^{\dagger}$ with $L_{i}=A_{i} \otimes B_{i}$, it is clear that the channel is non-entangling in the sense that it maps separable density matrices to separable density matrices. We then imposed a time-translation invariance assumption to write the time evolution under this map in Lindblad form. To do this, one expands the Krauss operators to lowest order in $d t$ as

$$
L_{0}=1-i H d t+K d t, \quad L_{i}=E_{i} \sqrt{d t}, \quad K=\frac{1}{2} \sum_{i \geq 1} L_{i}^{\dagger} L_{i} .
$$

We then applied the Krauss separability condition $L_{i}=A_{i} \otimes B_{i}$ to this expression to derive constraints on the Lindblad operators $E_{i}$.

The essential issue is that the separability criterion (c) need only be true in one particularly basis of Krauss operators, which is not generally the format given by the Kraus operators $L_{j}$ above. Any two sets of Krauss operators $\left\{L_{i}\right\},\left\{L_{j}^{\prime}\right\}$ related by unitary transformations $L_{i}=\sum_{j} U_{i j} L_{j}^{\prime}$ produce equivalent channels. These unitaries can be non-local, and thus a channel with a separable representation can be unitarily equivalent to a Lindblad form that does not satisfy separability in $H$ and $K$ in $L_{0}$. The problem is that computing the Lindblad representation of a channel (1) will generally require a basis

Published by the American Physical Society under the terms of the Creative Commons Attribution 4.0 International license. Further distribution of this work must maintain attribution to the author(s) and the published article's title, journal citation, and DOI. 
rotation to put one of the Krauss operators $L_{0}$ into the specific form $L_{0}=1+\mathcal{O}(d t)$. In particular, a given non-separable Krauss representation may require a non-local unitary rotation before admitting the expansion (1), and thus the constraints derived on the Lindblad operators in the paper are not generally correct.

Thus, we correct the theorem replacing statement (c) above with a more explicit assumption (c'), expressed directly at the level of the Lindblad equation:

(c') $L$ is generated by a separable Lindblad evolution: the differential time evolution $\rho(t)=L\left[\rho\left(t_{0}\right)\right]$ is given by

$$
\dot{\rho}=-i[H, \rho]+\sum_{i} E_{i} \rho E_{i}^{\dagger}-\frac{1}{2}\left\{E_{i}^{\dagger} E_{i}, \rho\right\}
$$

with the Hamiltonian $H$ and Lindblad operators $E_{i}$ all taking the form of product operators, and where $\sum_{i} E_{i}^{\dagger} E_{i}$ is a sum of operators acting on only $A$ or $B$.

Everything in the rest of the paper would then follow without change. (We also note that using such a Lindblad form already assumed the time-translation semigroup assumption (a) used in the paper, which would then be redundant). In particular, any such channel necessarily leads to a monotonically decaying atomic visibility.

This weakens, but does not trivialize, the basic result. It is possible to make a model which reproduces semi-classical gravitational interactions which takes the Lindblad form (2), and observation of the collapse and revival would rule this out. To see a concrete example, consider the experiment discussed in the paper, with a mechanical oscillator gravitationally coupled to an atom localized to one of two locations. We can construct a Lindbladian model with a purely stochastic gravitational force, that is, the force arises due to random application of impulses whose direction depends on the quantum state. Let $E_{0}=e^{-i \alpha x}|0\rangle\langle 0|$ and $E_{1}=e^{+i \alpha x}|1\rangle\langle 1|$, with $|0,1\rangle$ denoting the two atom locations and $\alpha$ a constant to be fixed shortly. These are product operators satisfying our sum rule. The evolution of the mechanical momentum under damping from $\left\{E_{0}, E_{1}\right\}$ with rate $\gamma$ is

$$
\dot{p}=\gamma \sum_{i} E_{i}^{\dagger} p E_{i}-\frac{1}{2}\left\{E_{i}^{\dagger} E_{i}, p\right\}=\gamma \alpha \sigma_{z}
$$

so setting the coefficient $\alpha=G_{N} m M \ell x_{0} / d^{3} \gamma$ produces the expected Newtonian force on the pendulum, dependent on the atomic position. In particular, this would give a "semiclassical" evolution like $\langle\dot{p}\rangle=\gamma \alpha\left\langle\sigma_{z}\right\rangle$. This evolution is incapable of generating revival dynamics [consistent with the theorem assuming (c')], and thus can be directly ruled out experimentally in the manner described in the paper.

However, the (correct) Lindblad version of the theorem is a weaker result: it does allow for construction of channels which are non-entangling yet still capable of producing the revival signals. We now give an explicit example of this. The example also suggests a way to close this loophole: such a channel requires adding certain extra noise terms to the evolution. The atomic interferometry experiment we originally proposed would be also capable of ruling these terms out, because these noise terms cause a partial loss of the revival signal.

Explicit example. We now construct a manifestly non-entangling channel which obeys the time-translation semigroup law, preserves $\sigma_{z}$, and still allows for partial collapse-and-revival dynamics. This is a measurement-and-feedback channel which uses only local operations. Concretely, consider two channels, one with Krauss operators

$$
K_{ \pm}=e^{ \pm i \beta \sigma_{z} \sqrt{d t}}[\cos (\alpha x \sqrt{d t}) \pm \sin (\alpha x \sqrt{d t})] / 2
$$

and the other with

$$
K_{ \pm}^{\prime}=e^{ \pm i \alpha x \sqrt{d t}}\left[\cos \left(\beta \sigma_{z} \sqrt{d t}\right) \pm \sin \left(\beta \sigma_{z} \sqrt{d t}\right)\right] / 2 .
$$

The coefficients $\alpha, \beta$ will be fixed shortly. These are both valid channels $\sum_{a} K_{a}^{\dagger} K_{a}=1, \sum_{a} K_{a}^{\prime \dagger} K_{a}^{\prime}=1$, and are manifestly separable. These arise by Ramsey-type measurements on either the qubit or oscillator, with outcomes \pm 1 , followed by feedback onto the other system. Concatenation of these channels thus forms a new channel $L$ with four Krauss operators $L_{a b}$, namely

$$
L_{a b}=K_{a}^{\prime} K_{b}
$$

These concatenated Krauss operators are again manifestly separable. Moreover, the channel clearly preserves $\sigma_{z}$, since the Krauss operators commute with $\sigma_{z}$. We just need to check if this channel generates Lindblad-form evolution. Notice 
that none of these Krauss operators have an expansion like that used for $L_{0}$ in (1). However, direct computation gives

$$
\begin{aligned}
d \rho & =L\left[\rho\left(t_{0}\right)\right]-\rho\left(t_{0}\right) \\
& =-2 i \alpha \beta\left[x \sigma_{z}, \rho\right] d t-\alpha^{2}\left(2 x \rho x-\left\{x^{2}, \rho\right\}\right) d t-\beta^{2}\left(2 \rho-2 \sigma_{z} \rho \sigma_{z}\right) d t+\mathcal{O}\left(d t^{3 / 2}\right),
\end{aligned}
$$

which we recognize as a Lindblad equation (2), with

$$
H=-2 \alpha \beta x \sigma_{z}, \quad E_{1}=i \sqrt{2} \alpha x, \quad E_{2}=\sqrt{2} \beta \sigma_{z} .
$$

We see that while the Lindblad operators $E_{i}$ are separable, the Hamiltonian term is not. This is despite the fact that, by construction, this channel is incapable of generating qubit-oscillator entanglement, since it admits a separable Krauss representation.

The Hamiltonian term (8) is precisely the one used in our paper to generate collapse-and-revival signals. See equation (2). Thus this non-entangling channel can at least partially mimic the signal expected from the full quantum channel where we have only the Hamiltonian term. The additional noise terms, however, would partially destroy the revival. Since the strength of these terms is linked to the Hamiltonian coupling, it may still be possible in general to rule out such models by observing a sufficiently complete revival. With our specific setup, the anomalous heating should be in the range of 1 phonon/Hz [? ? ], clearly observable above thermal noise in a high- $Q$ oscillator.

Summary and conclusions. The original claim in our paper was that observation of collapse-and-revival dynamics in the atomic interferometry experiment proposed there would rule out any model in which gravity does not generate entanglement. This conclusion was too strong; as explained above, the correct statement is that a revival only directly rules out any model with separable Lindblad operators. Although this does include a large class of models in which gravity is purely stochastic, it leaves open possible counter-examples constructed using LOCC measurement-and-feedback channels. We close by noting that such channels necessarily produce additional gravitational-strength noise [i.e. the $E_{1}, E_{2}$ operators in (8)], and could thus possibly be ruled out by the same experiment, but leave a detailed study of this idea to a future paper.

[1] K. Streltsov, J. S. Pedernales, and M. B. Plenio, (2021), arXiv:2111.04570 [quant-ph].

[2] D. Carney, H. Muller, and J. M. Taylor, (2021), arXiv:2111.04667 [quant-ph]. 